\title{
MULTIPLE EXISTENCE AND STABILITY OF STEADY-STATES FOR A PREY-PREDATOR SYSTEM WITH CROSS-DIFFUSION
}

\author{
KOUSUKE KUTO and YOSHIO YAMADA \\ Department of Mathematics, Waseda University \\ 3-4-1 Ohkubo, Shinjuku-ku, Tokyo 169-8555, Japan \\ E-mail:kuto@toki.waseda.jp,yamada@waseda.jp
}

\begin{abstract}
This article discusses a prey-predator system with cross-diffusion. We obtain multiple positive steady-state solutions of this system. More precisely, we prove that the set of positive steady-states possibly contains an $\mathrm{S}$ or $\supset$-shaped branch with respect to a bifurcation parameter in the large cross-diffusion case. Next we give some criteria on the stability of these positive steady-states. Furthermore, we find the Hopf bifurcation point on the steady-state solution branch in a certain case. Our method of analysis uses the idea developed by Du and Lou [6] and is based on the bifurcation theory and the Lyapunov-Schmidt reduction technique.
\end{abstract}

1. Introduction. In this article, we are concerned with the following Lotka-Volterra prey-predator model with cross-diffusion:

$$
(\mathrm{P}) \begin{cases}u_{t}=\Delta u+u(a-u-c v) & \text { in } \Omega \times(0, \infty), \\ \sigma v_{t}=\Delta[(1+\beta u) v]+v(b+d u-v) & \text { in } \Omega \times(0, \infty), \\ u=v=0 & \text { on } \partial \Omega \times(0, \infty), \\ u(\cdot, 0)=u_{0} \geq 0, \quad v(\cdot, 0)=v_{0} \geq 0 & \text { in } \Omega,\end{cases}
$$

where $\Omega$ is a bounded domain in $\boldsymbol{R}^{N}(N \geq 1)$ with smooth boundary $\partial \Omega ; \sigma, a, b, c, d$ are positive constants and $\beta \geq 0$ is the cross-diffusion coefficient. In $(\mathrm{P})$, unknown functions $u$ and $v$ represent the population densities of prey and predator species, respectively, which are interacting and migrating in the same habitat $\Omega$. In a certain kind of prey-predator

2000 Mathematics Subject Classification: Primary 35J65; Secondary 92D25.

Key words and phrases: cross-diffusion, steady-state, multiple existence, stability, Hopf bifurcation, Lyapunov-Schmidt reduction.

Research of the second author partially supported by Grant-in-Aid for Scientific Research (No.12640224), The Ministry of Education, Culture, Sports, Science and Technology, Japan and by Waseda University Grant for Special Research Projects 2002A-074.

The paper is in final form and no version of it will be published elsewhere. 
relationships, a great number of prey species form a huge group to protect themselves from the attack of predator. So we assume that the population pressure due to the high density of prey induces the diffusion of the form $\beta \Delta(u v)$ in the second equation. The boundary condition means that the habitat $\Omega$ is surrounded by a hostile environment. See also the monograph of Okubo and Levin [23] for the biological background. It should be noted that the local solvability of $(\mathrm{P})$ has been established by Amann [1], where a wide class of quasilinear parabolic systems is discussed. According to his result, $(\mathrm{P})$ has a unique local solution $(u, v)$ provided $\left(u_{0}, v_{0}\right) \in W_{0}^{1, p}(\Omega) \times W_{0}^{1, p}(\Omega)$ for $p>N$, and moreover, $u \geq 0, v \geq 0$ for all $(x, t) \in \Omega \times[0, T)$, where $T$ is the maximal existence time of $(u, v)$. Recently, Kuiper and Le [9] have found the global attractor for a class of triangular cross diffusion systems involving $(\mathrm{P})$.

System (P) originates from the competition population model with cross-diffusion proposed by Shigesada, Kawasaki and Teramoto [26]. Since their pioneer work, many mathematicians have discussed such cross-diffusion systems (primary on competition models) from various view-points, e.g., steady-state problems $([7,15,16,17,19,20,21,22,25])$ and the global existence of time-dependent solutions $([3,4,9,12,13,18,27])$. Nevertheless their all works, concerning cross-diffusion systems, many problems still remain open now. In particular, it is very difficult to know the detailed structure of the steady-state solution set (e.g., the number, the stability or the shape of steady-states) to cross-diffusion systems such as $(\mathrm{P})$.

Our aim in this article is to obtain the global bifurcation structure of positive steadystate solutions to $(\mathrm{P})$ in a special case when $\beta$ is sufficiently large. Regarding $a$ as a bifurcation parameter, we set

$$
\mathcal{S}:=\{(u, v, a):(u, v) \text { is a positive steady-state solution of }(\mathrm{P})\} .
$$

Assuming that $\beta$ is large and some coefficient conditions, we show that $\mathcal{S}$ contains an $\mathrm{S}$ or $\supset$-shaped curve with respect to the bifurcation parameter $a$. Then $(\mathrm{P})$ admits two or three positive steady-state solutions if $a$ belongs to suitable ranges. This result implies a great contrast to the linear diffusion case $(\beta=0)$, where the uniqueness of positive steady-states is obtained by López-Gómez and Pardo [14] if the spatial dimension is one. Our method of analysis uses the idea developed by Du and Lou [6] and is based on the bifurcation theory and the Lyapunov-Schmidt reduction procedure. If $\beta$ is large and both of $b-\lambda_{1}$ and $\lambda_{1}-d / \beta$ are small positives, this reduction enables us to find a relationship to a suitable limiting problem. Further, we can get the solution set with an explicit expression of the limiting problem. Making use of the perturbation theory developed in [6], we will depict an $\mathrm{S}$ or $\supset$-shaped curve of $\mathcal{S}$ near the limiting solution set.

In Section 2 we will discuss such multiple existence of steady-state solutions. In Section 3 , we will give some criteria on the stability of the positive steady-states. Furthermore, we will find the Hopf bifurcation point on the $\mathrm{S}$ or $\supset$-shaped solution set if $\sigma$ is sufficiently large.

Throughout the article, the usual norms of the spaces $L^{p}(\Omega)$ for $p \in[1, \infty)$ and $C(\bar{\Omega})$ are defined by

$$
\|u\|_{p}:=\left(\int_{\Omega}|u(x)|^{p} d x\right)^{1 / p} \text { and }\|u\|_{\infty}:=\max _{x \in \bar{\Omega}}|u(x)| .
$$


In particular, we simply write $\|u\|$ instead of $\|u\|_{2}$. Furthermore, we will denote by $\Phi$ a unique positive solution of

$$
-\Delta \Phi=\lambda_{1} \Phi \text { in } \Omega, \quad \Phi=0 \text { on } \partial \Omega, \quad\|\Phi\|=1,
$$

where $\lambda_{1}$ is the least eigenvalue of $-\Delta$ with the homogeneous Dirichlet boundary condition on $\partial \Omega$.

\section{Multiple existence of positive steady-state solutions.}

2.1. Main result. It is well known that the problem

$$
\Delta u+u(a-u)=0 \text { in } \Omega, \quad u=0 \text { on } \partial \Omega
$$

has a unique positive solution $\theta_{a}$ if $a>\lambda_{1}$; moreover, $a \in\left[\lambda_{1}, \infty\right) \rightarrow \theta_{a} \in C(\bar{\Omega})$ is continuous and strictly increasing function. It is possible to show that $(\mathrm{P})$ has two semitrivial steady-state solutions

$$
(u, v)=\left(\theta_{a}, 0\right) \text { for } a>\lambda_{1} \quad \text { and } \quad(u, v)=\left(0, \theta_{b}\right) \text { for } b>\lambda_{1}
$$

in addition to the trivial solution $(u, v)=(0,0)$.

Our result asserts that $\mathcal{S}$ contains a bounded $\mathrm{S}$ or $\supset$-shaped branch, which connects the above two semitrivial solutions, in a certain case:

Theorem 2.1. Assume $\beta b>\beta \lambda_{1}>d$. For any $c>0$, there exist a large number $M$ and an open set

$$
O=O(c) \subset\left\{(\beta, b, d): \beta \geq M, 0<\lambda_{1}-d / \beta, b-\lambda_{1} \leq M^{-1}\right\}
$$

such that if $(\beta, b, d) \in O$, then $\mathcal{S}$ contains a bounded smooth curve

$$
\Gamma=\left\{(u(r), v(r), a(r)) \in C^{1}(\bar{\Omega}) \times C^{1}(\bar{\Omega}) \times\left(\lambda_{1}, \infty\right), r \in(0, C)\right\}
$$

which possesses the following properties:

(i) $(u(0), v(0))=\left(0, \theta_{b}\right), \quad a(0)>\lambda_{1}, \quad a^{\prime}(0)>0$;

(ii) $(u(C), v(C))=\left(\theta_{a(C)}, 0\right), \quad a(C)>\lambda_{1}$;

(iii) $a(r)$ attains a strict local maximum in $(0, C)$. Additionally, there exists an open set $O^{\prime} \subset O$ such that, if $(\beta, b, d) \in O^{\prime}$, then $a(r)$ attains a strict local minimum in $(0, C)$.

It is noted that we can find an unbounded S-shaped branch of $\mathcal{S}$, under another coefficient assumption [11, Theorem 1.2].

2.2. Sketch of the proof of Theorem 2.1. In (P), we employ the following change of variables:

$$
a=\lambda_{1}+\varepsilon a_{1}, \quad b=\lambda_{1}+\varepsilon b_{1}, \quad d / \beta=\lambda_{1}-\varepsilon \tau, \quad \beta=\gamma / \varepsilon, \quad u=\varepsilon w, \quad(1+\beta u) v=\varepsilon z .
$$

Here $a_{1}, b_{1}, \tau$ are positive constants. Furthermore, $\varepsilon$ is a small positive constant, thus $\gamma$ is also a positive constant. In what follows, we will mainly discuss the case when $\beta$ is large and both of $b-\lambda_{1}$ and $\lambda_{1}-d / \beta$ are small positives. We note that $a_{1}$ plays a role of a 
bifurcation parameter. By (2.1), a pair of new unknown functions $(w, z)$ satisfies

$$
(\mathrm{PP}) \begin{cases}w_{t}=\Delta w+\lambda_{1} w+\varepsilon f\left(w, z, a_{1}\right) & \text { in } \Omega \times(0, \infty), \\ \sigma\left[-\frac{\gamma z}{(1+\gamma w)^{2}} w_{t}+\frac{z_{t}}{1+\gamma w}\right]=\Delta z+\lambda_{1} z+\varepsilon g(w, z) & \text { in } \Omega \times(0, \infty), \\ w=z=0 & \text { on } \partial \Omega \times(0, \infty), \\ w(\cdot, 0)=u_{0} / \varepsilon, \quad z(\cdot, 0)=\left(1+\beta u_{0}\right) v_{0} / \varepsilon & \text { in } \Omega,\end{cases}
$$

where

$$
\left\{\begin{array}{l}
f\left(w, z, a_{1}\right):=w\left(a_{1}-w-\frac{c z}{1+\gamma w}\right), \\
g(w, z):=\frac{z}{1+\gamma w}\left(b_{1}-\tau \gamma w-\frac{z}{1+\gamma w}\right) .
\end{array}\right.
$$

Here we note that positivity of solutions of $(\mathrm{P})$ assures that of solutions of $(\mathrm{PP})$. The steady-state problem associated with $(\mathrm{PP})$ is reduced to the following semilinear elliptic equations:

$$
\begin{cases}\Delta w+\lambda_{1} w+\varepsilon f\left(w, z, a_{1}\right)=0 & \text { in } \Omega, \\ \Delta z+\lambda_{1} z+\varepsilon g(w, z)=0 & \text { in } \Omega, \\ w=z=0 & \text { on } \partial \Omega .\end{cases}
$$

By virtue of (2.1), it is easy to see that (2.3) has two semitrivial solutions

$$
(w, z)=\left(\varepsilon^{-1} \theta_{\lambda_{1}+\varepsilon a_{1}}, 0\right), \quad(w, z)=\left(0, \varepsilon^{-1} \theta_{\lambda_{1}+\varepsilon b_{1}}\right)
$$

in addition to the trivial solution. For the Lyapunov-Schmidt reduction, we will give a similar framework to that of Du and Lou [6]. For $p>N$, we define two Banach spaces

$$
\left\{\begin{array}{l}
X:=\left[W^{2, p}(\Omega) \cap W_{0}^{1, p}(\Omega)\right] \times\left[W^{2, p}(\Omega) \cap W_{0}^{1, p}(\Omega)\right], \\
Y:=L^{p}(\Omega) \times L^{p}(\Omega) .
\end{array}\right.
$$

We note that $X \subset C^{1}(\bar{\Omega}) \times C^{1}(\bar{\Omega})$ by the Sobolev embedding theorem. Define mappings $H: X \rightarrow Y$ and $B: X \times \boldsymbol{R} \rightarrow Y$ by

$$
\left\{\begin{array}{l}
H(w, z):=\left(\Delta w+\lambda_{1} w, \Delta z+\lambda_{1} z\right) \\
B\left(w, z, a_{1}\right):=\left(f\left(w, z, a_{1}\right), g(w, z)\right)
\end{array}\right.
$$

Then (2.3) is equivalent to the equation

$$
H(w, z)+\varepsilon B\left(w, z, a_{1}\right)=0 .
$$

Let $X_{1}$ and $Y_{1}$ be the $L^{2}$-orthogonal complements of span $\{(\Phi, 0),(0, \Phi)\}$ in $X$ and $Y$, respectively. Let $P: X \rightarrow X_{1}$ and $Q: Y \rightarrow Y_{1}$ represent $L^{2}$-orthogonal projections. Thus a pair of unknown functions $(w, z) \in X$ is decomposed as

$$
(w, z)=(r, s) \Phi+\boldsymbol{u}, \quad \boldsymbol{u}=P(w, z) .
$$

Since $H((r, s) \Phi)=0$ and $(I-Q) H\left(X_{1}\right)=0,(2.5)$ is consequently reduced to

$$
Q H(\boldsymbol{u})+\varepsilon Q B\left((r, s) \Phi+\boldsymbol{u}, a_{1}\right)=0
$$

and

$$
(I-Q) B\left((r, s) \Phi+\boldsymbol{u}, a_{1}\right)=0 .
$$


The Lyapunov-Schmidt reduction procedure leads us to the next lemma:

Lemma 2.2. For any $C>0$, there exist a neighborhood $N_{0}$ of the set

$$
\left\{\left(w, z, a_{1}, \varepsilon\right)=\left(r \Phi, s \Phi, a_{1}, 0\right) \in X \times \boldsymbol{R}^{2}:|r|,|s|,\left|a_{1}\right| \leq C\right\}
$$

and a positive constant $\varepsilon_{0}$ such that all solutions of (2.6) in $N_{0}$ are given by

$$
\left\{\left((r, s) \Phi+\varepsilon \boldsymbol{U}\left(r, s, a_{1}, \varepsilon\right), a_{1}, \varepsilon\right):|r|,|s|,\left|a_{1}\right| \leq C+\varepsilon_{0},|\varepsilon| \leq \varepsilon_{0}\right\} .
$$

with a smooth $X_{1}$-valued function $\boldsymbol{U}$. Then

$$
\left(w, z, a_{1}, \varepsilon\right)=\left((r, s) \Phi+\varepsilon \boldsymbol{U}\left(r, s, a_{1}, \varepsilon\right), a_{1}, \varepsilon\right)
$$

becomes a solution of (2.5), or equivalently (2.3), in $N_{0}$ if and only if

$$
F^{\varepsilon}\left(r, s, a_{1}\right) \Phi:=(I-Q) B\left((r, s) \Phi+\varepsilon \boldsymbol{U}\left(r, s, a_{1}, \varepsilon\right), a_{1}\right)=0 .
$$

See [11] for the proof of Lemma 2.2. Since $(I-Q)(u, v)=\left(\int_{\Omega} u \Phi d x, \int_{\Omega} v \Phi d x\right) \Phi$, it follows from (2.2) and (2.4) that

$$
\begin{aligned}
F^{0}\left(r, s, a_{1}\right) & =\left(\int_{\Omega} f\left(r \Phi, s \Phi, a_{1}\right) \Phi, \int_{\Omega} g(r \Phi, s \Phi) \Phi\right) \\
& =\left(\begin{array}{c}
r\left(a_{1}-r\|\Phi\|_{3}^{3}-c s \int_{\Omega} \frac{\Phi^{3}}{1+\gamma r \Phi}\right) \\
s\left\{b_{1}-\left(b_{1}+\tau\right) \gamma r \int_{\Omega} \frac{\Phi^{3}}{1+\gamma r \Phi}-s \int_{\Omega} \frac{\Phi^{3}}{(1+\gamma r \Phi)^{2}}\right\}
\end{array}\right) .
\end{aligned}
$$

Thus Ker $F^{0}$ is the union of the following four sets:

$$
\begin{aligned}
& \mathcal{L}_{0}=\left\{\left(0,0, a_{1}\right): a_{1} \in \boldsymbol{R}\right\}, \\
& \mathcal{L}_{1}=\left\{\left(a_{1} /\left\|\phi_{1}\right\|_{3}^{3}, 0, a_{1}\right): a_{1} \in \boldsymbol{R}\right\}, \\
& \mathcal{L}_{2}=\left\{\left(0, b_{1} /\left\|\phi_{1}\right\|_{3}^{3}, a_{1}\right): a_{1} \in \boldsymbol{R}\right\}, \\
& \mathcal{L}_{p}=\{(r, \varphi(\gamma r), \psi(r)): r \in \boldsymbol{R}\},
\end{aligned}
$$

where

$$
\left\{\begin{array}{l}
\varphi(r)=\left[b_{1}-\left(b_{1}+\tau\right) r \int_{\Omega} \frac{\Phi^{3}}{1+r \Phi}\right]\left(\int_{\Omega} \frac{\Phi^{3}}{(1+r \Phi)^{2}}\right)^{-1}, \\
\psi(r)=r\|\Phi\|_{3}^{3}+c \varphi(\gamma r) \int_{\Omega} \frac{\Phi^{3}}{1+\gamma r \Phi}
\end{array}\right.
$$

We note that $\mathcal{L}_{p} \cap{\overline{\boldsymbol{R}_{+}}}^{3}$ means the limiting set of positive solutions of $(2.3)$ as $\varepsilon \rightarrow 0$. Indeed the following proposition holds true:

Proposition 2.3. For a sufficiently large $A_{1}>0$, there exist $\varepsilon_{0}>0$ and a family of smooth curves

$$
\left\{\left(r(\xi, \varepsilon), s(\xi, \varepsilon), a_{1}(\xi, \varepsilon)\right) \in \boldsymbol{R}_{+}^{3}:(\xi, \varepsilon) \in\left(0, C_{\varepsilon}\right) \times\left(0, \varepsilon_{0}\right)\right\}
$$

such that for each fixed $\varepsilon \in\left(0, \varepsilon_{0}\right]$, all positive solutions of $(2.3)$ with $a_{1} \in\left(0, A_{1}\right]$ can be parametrized as

$$
\begin{array}{r}
\Gamma^{\varepsilon}=\left\{\left(w(\xi, \varepsilon), z(\xi, \varepsilon), a_{1}(\xi, \varepsilon)\right)=\left((r, s) \Phi+\varepsilon \boldsymbol{U}\left(r, s, a_{1}, \varepsilon\right), a_{1}\right):\right. \\
\left.\left(r, s, a_{1}\right)=\left(r(\xi, \varepsilon), s(\xi, \varepsilon), a_{1}(\xi, \varepsilon)\right) \text { for } \xi \in\left(0, C_{\varepsilon}\right)\right\}
\end{array}
$$


and $\left(r(\xi, 0), s(\xi, 0), a_{1}(\xi, 0)\right)=(\xi, \varphi(\gamma \xi), \psi(\xi)), r(0, \varepsilon)=0$. Here $C_{\varepsilon}>0$ depends continuously on $\varepsilon \in\left[0, \varepsilon_{0}\right]$. Furthermore,

$$
w\left(C_{\varepsilon}, \varepsilon\right)>0 \text { in } \Omega \text { and } z\left(C_{\varepsilon}, \varepsilon\right) \equiv 0 .
$$

The above proposition implies that if $\varepsilon>0$ is sufficiently small, then $\Gamma^{\varepsilon}$ forms a positive solution branch near the curve $\{(r \Phi, \varphi(\gamma r) \Phi, \psi(r)): 0<r<C\}$. So it is important to study the profile of $\mathcal{L}_{p}$. By virtue of $(2.8)$,

$$
(0, \varphi(0), \psi(0))=\left(0, b_{1} /\|\Phi\|_{3}^{3}, c b_{1}\right) \in \mathcal{L}_{2} .
$$

It is easy to find a positive constant $r_{0}=r_{0}\left(\tau / b_{1}\right)$ such that

$$
\left\{\begin{array}{l}
\varphi(r)>0 \text { for } r \in\left[0, r_{0}\right), \\
\varphi(r)<0 \text { for } r \in\left(r_{0}, \infty\right)
\end{array}\right.
$$

Thus it follows that

$$
\left(r_{0} / \gamma, \varphi\left(r_{0}\right), \psi\left(r_{0} / \gamma\right)\right)=\left(r_{0} / \gamma, 0, r_{0}\|\Phi\|_{3}^{3} / \gamma\right) \in \mathcal{L}_{1}
$$

We note that $C_{\varepsilon}$ stated in Proposition 2.3 satisfies $C_{0}=r_{0} / \gamma$. Additionally the next lemma gives profiles of $\psi(r)$ in the interval of $\{r>0: \varphi(\gamma r)>0\}$ when $\tau$ is close to 0 and $\gamma$ is sufficiently large.

LEMMA 2.4. There exist positive constants $\tilde{\tau}=\tilde{\tau}\left(c, b_{1}\right)$ and $\tilde{\gamma}=\tilde{\gamma}\left(c, b_{1}\right)$ such that if $(\tau, \gamma) \in(0, \tilde{\tau}] \times[\tilde{\gamma}, \infty)$, then $\psi^{\prime}(0)>0$ and $\psi(r)$ achieves a strict local maximum in $\left(0, r_{0} / \gamma\right)$. Furthermore, there exists a continuous function $\hat{\gamma}(\tau)$ in $(0, \tilde{\tau}]$ satisfying

$$
\tilde{\gamma}<\hat{\gamma}(\tau) \text { for all } \tau \in(0, \tilde{\tau}] \text { and } \lim _{\tau \downarrow 0} \hat{\gamma}(\tau)=\infty
$$

and that, if $\gamma \in[\tilde{\gamma}, \hat{\gamma}(\tau))$ for $\tau \in(0, \tilde{\tau}]$, then $\psi(r)$ attains a strict local minimum in $\left(0, r_{0} / \gamma\right)$.

From Proposition 2.3 and Lemma 2.4, one can see the following proposition.

Proposition 2.5. Suppose that $(\tau, \gamma) \in(0, \tilde{\tau}] \times[\tilde{\gamma}, \infty)$ and that $\varepsilon>0$ is small enough. Then the positive solution set of (2.3) contains a bounded smooth curve

$$
\Gamma^{\varepsilon}=\left\{\left(w(\xi), z(\xi), a_{1}(\xi)\right) \in X \times \boldsymbol{R}: \xi \in\left(0, C_{\varepsilon}\right)\right\},
$$

which possesses the following properties:

(i) $(w(0), z(0))=\left(0, \varepsilon^{-1} \theta_{\lambda_{1}+\varepsilon b_{1}}\right), a_{1}(0)>0, a_{1}^{\prime}(0)>0$;

(ii) $\left(w\left(C_{\varepsilon}\right), z\left(C_{\varepsilon}\right)\right)=\left(\varepsilon^{-1} \theta_{\lambda_{1}+\varepsilon a_{1 *}}, 0\right), a_{1 *}:=a_{1}\left(C_{\varepsilon}\right)>0$;

(iii) $a_{1}(\xi)$ attains a strict local maximum in $\left(0, C_{\varepsilon}\right)$. In particular, if $\gamma \in[\tilde{\gamma}, \hat{\gamma}(\tau))$ for $\tau \in(0, \tilde{\tau}]$, then $a_{1}(\xi)$ attains a strict local minimum in $\left(0, C_{\varepsilon}\right)$.

With use of (2.1), Theorem 2.1 immediately follows from Proposition 2.5. Actually, for small $\varepsilon>0$, open sets stated in Theorem 2.1 are expressed as

$$
\begin{aligned}
& O=\left\{(\beta, b, d)=\left(\gamma / \varepsilon, \lambda_{1}+\varepsilon b_{1},\left(\lambda_{1}+\varepsilon \tau\right) \gamma / \varepsilon\right):(\tau, \gamma) \in(0, \tilde{\tau}) \times(\tilde{\gamma}, \infty)\right\}, \\
& O^{\prime}=\left\{(\beta, b, d)=\left(\gamma / \varepsilon, \lambda_{1}+\varepsilon b_{1},\left(\lambda_{1}+\varepsilon \tau\right) \gamma / \varepsilon\right):(\tau, \gamma) \in(0, \tilde{\tau}) \times(\tilde{\gamma}, \hat{\gamma}(\tau))\right\} .
\end{aligned}
$$

We refer to [11] for the complete proofs. 


\section{Stability analysis}

3.1. Main results. In this section, we will discuss the stability of steady-state solutions on $\Gamma$ obtained in Theorem 2.1. Before stating our stability results, we need to divide $\Gamma$ at every turning point with respect to $a$. In case $(\beta, b, d) \in O$, let

$$
0<r_{1}<r_{2}<\cdots<r_{k-1}<C
$$

be all strict local maximum or minimum points of $a(r)$. Since $a^{\prime}(0)>0$ (see Theorem $2.1), r_{2 j-1}(j=1,2, \ldots,[k / 2])$ are strict local maximum points, and $r_{2 j}(j=1,2, \ldots,[(k-$ 1)/2]) are strict local minimum points. For each $1 \leq i \leq k$, we set

$$
\Gamma_{i}:=\left\{(u(r), v(r), a(r)) \in \Gamma: r \in\left(r_{i-1}, r_{i}\right)\right\},
$$

where $r_{0}:=0$ and $r_{k}:=C$.

We are ready to state stability results. In case when $\sigma$ is sufficiently small, we can deduce that the stability of steady-states on $\Gamma$ changes only at the turning points, and moreover, we can know whether each solution on $\Gamma_{i}$ is asymptotically stable or not:

TheOREM 3.1. For almost every $(\beta, b, d) \in O$, there exists a small positive constant $\delta$ such that if $\sigma \leq \delta$, then all steady-state solutions on $\Gamma_{2 j-1}(j=1,2, \ldots,[(k+1) / 2])$ are asymptotically stable in the topology of $X$, while all steady-state solutions on $\Gamma_{2 j}(j=$ $1,2, \ldots,[k / 2])$ are unstable.

In the above case, we remark that $(u(0), v(0))=\left(0, \theta_{b}\right)$ and $(u(C), v(C))=\left(\theta_{a(C)}, 0\right)$ by Theorem 2.1. So Theorem 3.1 implies that stable positive steady-states bifurcate from the semitrivial solution $\left(0, \theta_{b}\right)$, the stability on $\Gamma$ changes at every turning point with respect to $a$, and moreover $\Gamma$ connects the other semitrivial solution $\left(\theta_{a(C)}, 0\right)$. On the other hand, when $\sigma$ becomes large enough, we can find the Hopf bifurcation point on $\Gamma_{1}$; so that, time-periodic solutions of $(\mathrm{P})$ appear from the point:

Theorem 3.2. For any $(\beta, b, d) \in O$, there exists a large positive $D$ such that if $\sigma \geq D$, then the Hopf bifurcation occurs at some point $\left(u\left(r^{*}\right), v\left(r^{*}\right), a\left(r^{*}\right)\right) \in \Gamma_{1}$. In this case, there exists a periodic solution of $(\mathrm{P})$ if a lies in a neighborhood of $a\left(r^{*}\right)$ with $a>a\left(r^{*}\right)$.

3.2. Sketch of the proofs of Theorems 3.1 and 3.2. By the regularity of (2.1), the stability of a steady-state $\left(u^{*}, v^{*}\right)$ of $(\mathrm{P})$ coincides with that of the steady-state $\left(w^{*}, z^{*}\right)=$ $\left(u^{*} / \varepsilon,\left(1+\beta u^{*}\right) z^{*} / \varepsilon\right)$ of $(\mathrm{PP})$. So we will concentrate on the stability analysis for the steady-states on $\Gamma^{\varepsilon}$ given in Proposition 2.5. By Proposition 2.3, all positive steady-states of $(\mathrm{PP})$ with $a_{1} \in\left(0, A_{1}\right)$ are parametrized as

$$
\left.\Gamma^{\varepsilon}=\left\{\left(w(\xi, \varepsilon), z(\xi, \varepsilon), a_{1}(\xi, \varepsilon)\right): \xi \in\left(0, C_{\varepsilon}\right)\right)\right\}
$$

when $\varepsilon>0$ is sufficiently small. For each $\left(w(\xi, \varepsilon), z(\xi, \varepsilon), a_{1}(\xi, \varepsilon)\right) \in \Gamma^{\varepsilon}$, we define a linear operator $L(\xi, \varepsilon): X \rightarrow Y$ by

$$
L(\xi, \varepsilon)\left(\begin{array}{l}
h \\
k
\end{array}\right):=-H\left(\begin{array}{l}
h \\
k
\end{array}\right)-\varepsilon B_{(w, z)}\left(w(\xi, \varepsilon), z(\xi, \varepsilon), a_{1}(\xi, \varepsilon)\right)\left(\begin{array}{l}
h \\
k
\end{array}\right),
$$

where $H, B$ are mappings defined by $(2.4)$ and $B_{(w, z)}$ denotes the Fréchet derivative of 
$B$ with respect to $(w, z)$. Furthermore, in view of the left hand side of $(\mathrm{PP})$, we set

$$
J(\xi, \varepsilon):=\left[\begin{array}{cc}
1 & 0 \\
-\frac{\sigma \gamma z(\xi, \varepsilon)}{(1+\gamma w(\xi, \varepsilon))^{2}} & \frac{\sigma}{1+\gamma w(\xi, \varepsilon)}
\end{array}\right] .
$$

Then the linearized eigenvalue problem associated with $(w(\xi, \varepsilon), z(\xi, \varepsilon))$ is given by

$$
L(\xi, \varepsilon)\left(\begin{array}{l}
h \\
k
\end{array}\right)=\mu J(\xi, \varepsilon)\left(\begin{array}{l}
h \\
k
\end{array}\right) \text {. }
$$

In this subsection, we study the linearized stability of steady-states on $\Gamma^{\varepsilon}$ by the spectral analysis for (3.1). Put

$$
\rho(\xi, \varepsilon):=\{\mu \in \boldsymbol{C}:(3.1) \text { has no solution except for } h=k=0\} .
$$

We begin with the following lemma for proofs.

Lemma 3.3. Suppose that $\varepsilon>0$ is sufficiently small. Then there exist positive constants $\kappa_{1}, \omega$ independent of $(\xi, \varepsilon)$ such that $-\rho(\xi, \varepsilon) \supset\left\{z \in C:|z| \geq \kappa_{1}\right.$ and $\left.|\arg z| \leq \pi / 2+\omega\right\}$. On the other hand, all eigenvalues $\left\{\mu_{i}(\xi, \varepsilon)\right\}_{i=1}^{\infty}$ (counting multiplicity) of (3.1) satisfy

$$
\lim _{\varepsilon \downarrow 0} \mu_{1}(\xi, \varepsilon)=\lim _{\varepsilon \downarrow 0} \mu_{2}(\xi, \varepsilon)=0
$$

and

$$
\operatorname{Re} \mu_{i}(\xi, \varepsilon)>\kappa_{2} \text { for all } i \geq 3 \text { and } \xi \in\left(0, C_{\varepsilon}\right)
$$

for some positive constant $\kappa_{2}$ independent of $(\xi, \varepsilon)$.

Proof. It follows from Proposition 2.3 that for any fixed $\xi \in\left(0, C_{\varepsilon}\right)$

$$
\lim _{\varepsilon \downarrow 0}\left(w(\xi, \varepsilon), z(\xi, \varepsilon), a_{1}(\xi, \varepsilon)\right)=(\xi \Phi, \varphi(\gamma \xi) \Phi, \psi(\xi)) \text { in } C^{1}(\bar{\Omega}) \times C^{1}(\bar{\Omega}) \times \boldsymbol{R} .
$$

Thus letting $\varepsilon \downarrow 0$ in (3.1), we have

$$
\begin{cases}-\Delta h-\lambda_{1} h=\mu h & \text { in } \Omega, \\ -\Delta k-\lambda_{1} k=\sigma \mu\left[-\frac{\gamma \varphi(\gamma \xi) \Phi}{(1+\gamma \xi \Phi)^{2}} h+\frac{k}{1+\gamma \xi \Phi}\right] & \text { in } \Omega, \\ h=k=0 & \text { on } \partial \Omega .\end{cases}
$$

Clearly, $\mu=0$ is a double eigenvalue of (3.3). If $h \not \equiv 0$, then each eigenvalue of (3.3) is real and nonnegative by the first equation. If $h \equiv 0$, we are led to the same result by the second equation. Consequently we see all eigenvalues of (3.3) are real and nonnegative. From this fact, we can obtain all assertions of Lemma 3.3 with the aid of the perturbation theory by T. Kato [8, Chapter 8].

We note that all eigenvalues $\left\{\mu_{i}(\xi, \varepsilon)\right\}$ form a symmetric set with respect to the real axis in the complex space $\boldsymbol{C}$. Then $\mu_{1}(\xi, \varepsilon)$ and $\mu_{2}(\xi, \varepsilon)$ (with (3.2)) satisfy the following properties (i) or (ii);

(i) both of $\mu_{1}(\xi, \varepsilon)$ and $\mu_{2}(\xi, \varepsilon)$ are real numbers;

(ii) $\mu_{1}(\xi, \varepsilon)$ is a complex conjugate of $\mu_{2}(\xi, \varepsilon)$.

In what follows, we assume that $\mu_{1}(\xi, \varepsilon) \leq \mu_{2}(\xi, \varepsilon)$ in case (i), and $\operatorname{Im} \mu_{1}(\xi, \varepsilon) \geq \operatorname{Im} \mu_{2}(\xi, \varepsilon)$ in case (ii). 
Definition 3.1 (Linearized stability). A steady-state $(w(\xi, \varepsilon), z(\xi, \varepsilon))$ of $(\mathrm{PP})$ is called linearly stable if $\operatorname{Re} \mu_{1}(\xi, \varepsilon)>0$. If $\operatorname{Re} \mu_{1}(\xi, \varepsilon)<0$, then it is called linearly unstable.

We define matrices $K(r)$ and $M(r)$ by

$$
\begin{aligned}
& K(r)=\left[\begin{array}{cc}
1 & 0 \\
-\sigma \gamma \varphi(\gamma r) \int_{\Omega} \frac{\Phi^{3}}{(1+\gamma r \Phi)^{2}} & \sigma \int_{\Omega} \frac{\Phi^{2}}{1+\gamma r \Phi}
\end{array}\right], \\
& M(r)=-K(r)^{-1} F_{(r, s)}^{0}(r, \varphi(\gamma r), \psi(r))
\end{aligned}
$$

for the mapping $F^{0}$ defined by (2.7). To determine the sign of $\operatorname{Re} \mu_{1}(\xi, \varepsilon)$, the following lemma plays an important role.

LEMma 3.4. Let $\nu_{1}(r)$ and $\nu_{2}(r)$ be eigenvalues of $M(r)$ and satisfy $\operatorname{Re} \nu_{1}(r) \leq \operatorname{Re} \nu_{2}(r)$, $\operatorname{Im} \nu_{1}(r) \geq \operatorname{Im} \nu_{2}(r)$. Then for any $r \in\left(0, C_{0}\right)$,

$$
\lim _{(\xi, \varepsilon) \rightarrow(r, 0)} \frac{\mu_{i}(\xi, \varepsilon)}{\varepsilon}=\nu_{i}(r) \text { for } i=1,2 .
$$

Lemma 3.4 can be proved by taking $L^{2}$-inner product of $(3.1)$ with $\Phi$ and letting $\varepsilon \rightarrow 0$. See $[10]$ for details.

Lemma 3.5. Suppose that $\varepsilon>0$ is sufficiently small. Suppose further that $\xi \in\left(0, C_{\varepsilon}\right)$. Thus all zeros of $\mu_{1}(\xi, \varepsilon)$ coincide with all zeros of $\partial_{\xi} a_{1}(\xi, \varepsilon)$.

The above lemma asserts that the degeneracy of steady-states on $\Gamma^{\varepsilon}$ is equivalent to the criticality of $a_{1}(\xi, \varepsilon)$ with respect to $\xi$. The proof of Lemma 3.5 applies the perturbation theory for the Fredholm operator developed by Du and Lou [6, Theorem 3.13 and Appendix].

Since $\psi$ is analytic, $\psi^{\prime}$ possesses at most a finite number of zeros in $\left(0, C_{0}\right)$. Furthermore, by (2.8), any zero of $\psi^{\prime}$ must be a strictly critical point of $\psi$ for almost every $(\tau, \gamma) \in(0, \tilde{\tau}] \times[\tilde{\gamma}, \infty)$. For $\operatorname{such}(\tau, \gamma) \in(0, \tilde{\tau}] \times[\tilde{\gamma}, \infty)$ and sufficiently small $\varepsilon>0$, all zeros of $\partial_{\xi} a_{1}(\xi, \varepsilon)$ are denoted by

$$
0<\xi_{1}(\varepsilon)<\xi_{2}(\varepsilon)<\cdots<\xi_{k-1}(\varepsilon)<C_{\varepsilon} .
$$

That is,

$$
\left(w_{i}, z_{i}, a_{1}^{i}\right):=\left(w\left(\xi_{i}(\varepsilon), \varepsilon\right), z\left(\xi_{i}(\varepsilon), \varepsilon\right), a_{1}\left(\xi_{i}(\varepsilon), \varepsilon\right)\right) \in \Gamma^{\varepsilon} \quad(i=1,2, \ldots, k-1)
$$

are all turning points on $\Gamma^{\varepsilon}$ with respect to $a_{1}$. Here we remark that $\lim _{\varepsilon \downarrow 0} a_{1}(\cdot, \varepsilon)=\psi$ in $C^{2}\left(\left[0, C_{0}\right]\right)$ by Proposition 2.3 (see also the proof of [11, Lemma 5.3]). Additionally, for each $1 \leq i \leq k$ we set

$$
\Gamma_{i}^{\varepsilon}:=\left\{\left(\left(w(\xi, \varepsilon), z(\xi, \varepsilon), a_{1}(\xi, \varepsilon)\right): \xi \in\left(\xi_{i-1}(\varepsilon), \xi_{i}(\varepsilon)\right)\right\},\right.
$$

where $\xi_{0}(\varepsilon):=0$ and $\xi_{k}(\varepsilon)=C_{\varepsilon}$. This implies $\bigcup_{i=1}^{k} \Gamma_{i}^{\varepsilon}=\Gamma^{\varepsilon} \backslash \bigcup_{i=1}^{k-1}\left\{\left(w_{i}, z_{i}, a_{1}^{i}\right)\right\}$.

Lemma 3.6. For almost every $(\tau, \gamma) \in(0, \tilde{\tau}] \times[\tilde{\gamma}, \infty)$, there exist small positive constants $\delta, \varepsilon_{0}$ such that if $\sigma \leq \delta$ and $\varepsilon \leq \varepsilon_{0}$, then all steady-state solutions on $\Gamma_{2 j-1}^{\varepsilon}(j=$ $1,2, \ldots,[(k+1) / 2])$ are linearly stable, while all steady-state solutions on $\Gamma_{2 j}^{\varepsilon}(j=$ $1,2, \ldots,[k / 2])$ are linearly unstable. 
Proof. Taking the trace of $M(r)$, one can see

$$
\begin{aligned}
\nu_{1}(r)+\nu_{2}(r)= & \frac{\varphi(\gamma r)}{\sigma}\left[\int_{\Omega} \frac{\Phi^{3}}{(1+\gamma r \Phi)^{2}}\left(\int_{\Omega} \frac{\Phi^{2}}{1+\gamma r \Phi}\right)^{-1}-\sigma c \gamma r \int_{\Omega} \frac{\Phi^{4}}{(1+\gamma r \Phi)^{2}}\right] \\
& +r\|\Phi\|_{3}^{3}+c \gamma r \varphi(\gamma r) \int_{\Omega} \frac{\Phi^{3}}{1+\gamma r \Phi} \int_{\Omega} \frac{\Phi^{3}}{(1+\gamma r \Phi)^{2}}\left(\int_{\Omega} \frac{\Phi^{2}}{1+\gamma r \Phi}\right)^{-1} .
\end{aligned}
$$

We set $y_{1}(r):=\int_{\Omega} r \Phi^{4} /(1+r \Phi)^{2}$. Since $y_{1}(0)=0$ and $y_{1}(r)=O\left(r^{-1}\right)(r \rightarrow \infty), y_{1}(\hat{r})=$ $\sup _{r>0} y_{1}(r)$ for some $\hat{r}>0$. Then by (3.6), we obtain

$$
\begin{aligned}
\nu_{1}(r)+\nu_{2}(r) & \geq \frac{\varphi(\gamma r)}{\sigma}\left[\int_{\Omega} \frac{\Phi^{3}}{(1+\gamma r \Phi)^{2}}\left(\int_{\Omega} \frac{\Phi^{2}}{1+\gamma r \Phi}\right)^{-1}-\sigma c y_{1}(\gamma r)\right]+r\|\Phi\|_{3}^{3} \\
& >\frac{\varphi(\gamma r)}{\sigma}\left[\int_{\Omega} \frac{\Phi^{3}}{\left(1+\gamma C_{0} \Phi\right)^{2}}-\sigma c y_{1}(\hat{r})\right]+r\|\Phi\|_{3}^{3}
\end{aligned}
$$

for all $r \in\left[0, C_{0}\right]$. Therefore, it follows from $\varphi(\gamma r)>0\left(r \in\left[0, C_{0}\right)\right)$ that, if

$$
\sigma<\frac{1}{2 c y_{1}(\hat{r})} \int_{\Omega} \frac{\Phi^{3}}{\left(1+\gamma C_{0} \Phi\right)^{2}}
$$

then $\nu_{1}(r)+\nu_{2}(r)>0$ for all $r \in\left[0, C_{0}\right]$. Thus we can see by Lemma 3.4 that for sufficiently small $\varepsilon>0$,

$$
\mu_{1}(\xi, \varepsilon)+\mu_{2}(\xi, \varepsilon)>0 \text { for all } \xi \in\left[0, C_{\varepsilon}\right] .
$$

Hence (3.7) also implies $\operatorname{Re} \mu_{2}(\xi, \varepsilon)>0$ for all $\xi \in\left[0, C_{\varepsilon}\right]$. On the other hand, in view of (3.4), (2.7) and (2.8), direct calculations enable us to obtain

$$
\nu_{1}(r) \nu_{2}(r)=\operatorname{det} M(r)=\frac{r \varphi(\gamma r) \psi^{\prime}(r)}{\sigma} \int_{\Omega} \frac{\Phi^{3}}{(1+\gamma r \Phi)^{2}}\left(\int_{\Omega} \frac{\Phi^{2}}{1+\gamma r \Phi}\right)^{-1} .
$$

So $\operatorname{sign} \nu_{1}(r) \nu_{2}(r)=\operatorname{sign} \psi^{\prime}(r)$ for all $r \in\left(0, C_{0}\right)$. Let $r_{0} \in\left(0, C_{0}\right)$ be any fixed point. If $\psi^{\prime}\left(r_{0}\right)>0$, then Lemma 3.4 implies $\mu_{1}(\xi, \varepsilon) \mu_{2}(\xi, \varepsilon)>0$ if $(\xi, \varepsilon)$ is sufficiently near $\left(r_{0}, 0\right)$. Further, together with $(3.7)$, we obtain $\operatorname{Re} \mu_{1}(\xi, \varepsilon)>0$. Similarly if $\psi^{\prime}\left(r_{0}\right)<0$ and $(\xi, \varepsilon)$ is close to $\left(r_{0}, 0\right)$, then $\operatorname{Re} \mu_{1}(\xi, \varepsilon)<0$. Additionally it follows from Lemma 3.5 that $\mu_{1}(\xi, \varepsilon)=0$ if and only if $\xi=\xi_{i}(\varepsilon)$ for some $1 \leq i \leq k-1$ provided that $\varepsilon>0$ is sufficiently small. Since $\operatorname{Re} \mu_{2}(\xi, \varepsilon)>0$ for all $\xi \in\left[0, C_{\varepsilon}\right]$, consequently $\operatorname{Re} \mu_{1}(\xi, \varepsilon)=0$ holds if and only if $\xi=\xi_{i}(\varepsilon)$ for some $1 \leq i \leq k-1$. We now remark $\psi^{\prime}(0)>0$ if $(\tau, \gamma) \in(0, \tilde{\tau}] \times[\tilde{\gamma}, \infty)($ see $[11$, Lemma 4.1]). Therefore we obtain

$$
\left\{\begin{array}{l}
\operatorname{Re} \mu_{1}(\xi, \varepsilon)>0 \text { if }\left(w(\xi, \varepsilon), z(\xi, \varepsilon), a_{1}(\xi, \varepsilon)\right) \in \Gamma_{2 j-1}^{\varepsilon}, \\
\operatorname{Re} \mu_{1}(\xi, \varepsilon)<0 \text { if }\left(w(\xi, \varepsilon), z(\xi, \varepsilon), a_{1}(\xi, \varepsilon)\right) \in \Gamma_{2 j}^{\varepsilon} .
\end{array}\right.
$$

Thus the proof of Lemma 3.6 is complete.

By virtue of (2.1), we can derive the complete proof of Theorem 2.1 from Lemma 3.6. It should be noted that we use the linearized stability theory developed by Potier-Ferry [24] in the derivation. See [10] for details.

Proposition 3.7. For any $(\tau, \gamma) \in(0, \tilde{\tau}] \times[\tilde{\gamma}, \infty)$, there exist a large $D>0$ and a small $\varepsilon_{0}>0$ such that if $\sigma \geq D$ and $\varepsilon \leq \varepsilon_{0}$, then the Hopf bifurcation occurs at a certain point on $\Gamma_{1}^{\varepsilon}$. 
Proof. It suffices to find small positive numbers $\xi^{*}$ and $\varepsilon$ such that $\mu_{1}\left(\xi^{*}, \varepsilon\right), \mu_{2}\left(\xi^{*}, \varepsilon\right)$ form a pure imaginary pair and satisfy $\partial_{\xi} \operatorname{Re} \mu_{i}\left(\xi^{*}, \varepsilon\right)<0$ for $i=1,2$. We refer to Amann [2] for the abstract Hopf bifurcation theorem for strongly coupled parabolic equations.

Take $(\tau, \gamma) \in(0, \tilde{\tau}] \times[\tilde{\gamma}, \infty)$. Let $\nu_{1}(r)$ and $\nu_{2}(r)$ be eigenvalues of $M(r)$ defined by (3.4). We first remark that by $(3.8)$ and $\psi^{\prime}(0)>0$,

$$
\nu_{1}(r) \nu_{2}(r)>0 \text { for all } r \in\left(0, r_{1}\right)
$$

with some $r_{1}>0$. If we set

$$
y_{2}(r):=\int_{\Omega} \frac{\Phi^{4}}{(1+\gamma r \Phi)^{2}}-\int_{\Omega} \frac{\Phi^{3}}{1+\gamma r \Phi} \int_{\Omega} \frac{\Phi^{3}}{(1+\gamma r \Phi)^{2}}\left(\int_{\Omega} \frac{\Phi^{2}}{1+\gamma r \Phi}\right)^{-1}-\frac{\|\Phi\|_{3}^{3}}{c \gamma \varphi(\gamma r)}
$$

then (3.6) is rewritten as

$$
\nu_{1}(r)+\nu_{2}(r)=\frac{\varphi(\gamma r)}{\sigma}\left[\int_{\Omega} \frac{\Phi^{3}}{(1+\gamma r \Phi)^{2}}\left(\int_{\Omega} \frac{\Phi^{2}}{1+\gamma r \Phi}\right)^{-1}-\sigma c \gamma r y_{2}(r)\right] .
$$

Thus direct calculations imply

$$
\nu_{1}(0)+\nu_{2}(0)=\frac{1}{\sigma}, \quad \nu_{1}^{\prime}(0)+\nu_{2}^{\prime}(0)=\frac{1}{\sigma}\left(\tilde{C}-\sigma c \gamma y_{2}(0)\right)
$$

for some constant $\tilde{C}$ independent of $\sigma$. By Schwarz' inequality and $\|\Phi\|=1$, we see $\|\Phi\|_{4}^{4}>\|\Phi\|_{3}^{6}$. Thus it turns out that $y_{2}(0)=\|\Phi\|_{4}^{4}-\|\Phi\|_{3}^{6}-\|\Phi\|_{3}^{3}\left(c b_{1} \gamma\right)^{-1}>0$ if $\gamma$ is large enough. It follows from (3.10) that if $\sigma$ is sufficiently large, we can find a small positive number $r_{0} \in\left(0, r_{1}\right)$ such that

$$
\left\{\begin{array}{l}
\nu_{1}(r)+\nu_{2}(r)>0 \text { in }\left(0, r_{0}\right) \\
\nu_{1}\left(r_{0}\right)+\nu_{2}\left(r_{0}\right)=0 \text { and } \nu_{1}^{\prime}\left(r_{0}\right)+\nu_{2}^{\prime}\left(r_{0}\right)<0
\end{array}\right.
$$

We can find a certain $\left(\xi^{*}, \varepsilon\right)$ near $\left(r_{0}, 0\right)$, such that eigenvalues $\mu_{1}\left(\xi^{*}, \varepsilon\right), \mu_{2}\left(\xi^{*}, \varepsilon\right)$ are a pure imaginary pair and satisfy $\partial_{\xi} \operatorname{Re} \mu_{i}\left(\xi^{*}, \varepsilon\right)<0(i=1,2)$. In this part of the proof, we make use of Lemma 3.4 and Lyapunov-Schmidt reduction technique (see [10]). Therefore the Hopf bifurcation occurs at $\left(w\left(\xi^{*}, \varepsilon\right), z\left(\xi^{*}, \varepsilon\right), a_{1}\left(\xi^{*}, \varepsilon\right)\right)$, which belongs to $\Gamma_{1}^{\varepsilon}$ because $\xi^{*}$ is sufficiently small.

By (2.1), Proposition 3.7 immediately yields Theorem 3.2.

\section{References}

[1] H. Amann, Dynamic theory of quasilinear parabolic equations-II. Reaction-diffusion systems, Differential Integral Equations 3 (1990), 13-75.

[2] H. Amann, Hopf bifurcation in quasilinear reaction-diffusion systems, in: Delay Differential Equations and Dynamical Systems (Claremont, CA, 1990), S. Busenberg and M. Martelli (eds.), Lecture Notes in Math. 1475, Springer, Berlin, 1991, 53-63.

[3] Y. S. Choi, R. Lui and Y. Yamada, Existence of global solutions for the ShigesadaKawasaki-Teramoto model with weak cross-diffusion, Discrete Contin. Dynam. Systems 9 (2003), 1993-2000.

[4] Y. S. Choi, R. Lui and Y. Yamada, Existence of global solutions for the ShigesadaKawasaki-Teramoto model with strongly coupled cross-diffusion, Discrete Contin. Dynam. Systems 10 (2004), 719-730. 
[5] M. G. Crandall and P. H. Rabinowitz, Bifurcation, perturbation of simple eigenvalues, and linearized stability, Arch. Rational Mech. Anal. 52 (1973), 161-180.

[6] Y. Du and Y. Lou, S-shaped global bifurcation curve and Hopf bifurcation of positive solutions to a predator-prey model, J. Differential Equations 144 (1998), 390-440.

[7] Y. Kan-on, Stability of singularly perturbed solutions to nonlinear diffusion systems arising in population dynamics, Hiroshima Math. J. 23 (1993), 509-536.

[8] T. Kato, Perturbation Theory for Linear Operators, Springer, Berlin, 1966.

[9] K. H. Kuiper and D. Le, Global attractors for cross diffusion systems on domains of arbitrary dimension, preprint.

[10] K. Kuto, Stability of steady-state solutions to a prey-predator system with cross-diffusion, J. Differential Equations 197 (2004), 293-314.

[11] K. Kuto and Y. Yamada, Multiple coexistence states for a prey-predator system with crossdiffusion, J. Differential Equations 197 (2004), 315-348.

[12] D. Le, Cross diffusion systems on $n$ spatial dimension domains, Indiana Univ. Math. J. 51 (2002), 625-643.

[13] D. Le, L. V. Nguyen and T. T. Nguyen, Shigesada-Kawasaki-Teramoto model on higher dimensional domains, Electronic J. Differential Equations 72 (2003), 1-12.

[14] J. López-Gómez and R. Pardo, Existence and uniqueness of coexistence states for the predator-prey model with diffusion, Differential Integral Equations 6 (1993), 1025-1031.

[15] Y. Lou and W.-M. Ni, Diffusion, self-diffusion and cross-diffusion, J. Differential Equations 131 (1996), 79-131.

[16] Y. Lou and W.-M. Ni, Diffusion vs cross-diffusion: An elliptic approach, J. Differential Equations 154 (1999), 157-190.

[17] Y. Lou, W.-M. Ni and S. Yotsutani, On a limiting system in the Lotka-Volterra competition with cross-diffusion, Discrete Contin. Dynam. Systems 10 (2004), 435-458.

[18] Y. Lou, W.-M. Ni and Y. Wu, On the global existence of a cross-diffusion system, Discrete Contin. Dynam. Systems 4 (1998), 193-203.

[19] M. Mimura, Stationary pattern of some density-dependent diffusion system with competitive interaction-diffusion equations, Hiroshima Math. J. 11 (1981), 621-635.

[20] M. Mimura and K. Kawasaki, Spatial segregation in competitive interaction-diffusion equations, J. Theor. Biol. 9 (1980), 49-64.

[21] M. Mimura, Y. Nishiura, A. Tesei and T. Tsujikawa, Coexistence problem for two competing species models with density-dependent diffusion, Hiroshima Math. J. 14 (1984), 425-449.

[22] K. Nakashima and Y. Yamada, Positive steady states for prey-predator models with crossdiffusion, Adv. Differential Equations 6 (1996), 1099-1122.

[23] A. Okubo and L. A. Levin, Diffusion and Ecological Problems: Modern Perspective, 2nd ed., Interdisciplinary Appl. Math. 14, Springer, New York, 2001.

[24] M. Potier-Ferry, The linearization principle for the stability of solutions of quasilinear parabolic equations-I, Arch. Rational Mech. Anal. 77 (1981), 301-320.

[25] K. Ryu and I. Ahn, Positive steady-states for two interacting species models with linear self-cross diffusions, Discrete Contin. Dynam. Systems 9 (2003), 1049-1061.

[26] N. Shigesada, K.Kawasaki and E. Teramoto, Spatial segregation of interacting species, J. Theor. Biol. 79 (1979), 83-99.

[27] A. Yagi, Global solution to some quasilinear parabolic system in population dynamics, Nonlinear Analysis T.M.A. 21 (1993), 531-556. 